\title{
Aggregation and sedimentation in gas-fluidized beds of cohesive powders
}

\author{
A. Castellanos, J. M. Valverde, and M. A. S. Quintanilla \\ Departamento Electronica y Electromagnetismo, Universidad de Sevilla, Avenida Reina Mercedes s/n, 41012 Sevilla, Spain
}

(Received 12 March 2001; revised manuscript received 14 May 2001; published 24 September 2001)

\begin{abstract}
We present measurements on the settling velocity of gas-fluidized beds of fine cohesive powders. In the solidlike regime (solid volume fraction $\phi>\phi_{c}$ ) particles are static, sustained by enduring contacts. The settling is hindered by interparticle contacts and is a very slow process. In the fluidlike regime $\left(\phi<\phi_{c}\right)$ permanent contacts no longer exist, and the bed displays a diffusive dynamics. The interparticle adhesive force leads to the formation of particle aggregates, and for this reason the sedimentation velocity exceeds the predicted value by empirical or theoretical laws on the settling of individual particles. We use an extension of the Richardson-Zaki empirical law for the settling of aggregates in the fluidlike regime to fit the experimental data. Aggregates are characterized by the number of aggregated particles $N$ and by an effective radius $R$. The trend followed by these parameters with particle size is confirmed by direct visualization of the aggregates, and shows that cohesive effects become important when the adhesion force between particles is above particle weight. Results show that aggregates form open structures with a fractal dimension close to the predicted one in the diffusionlimited-aggregation model $(D=2.5)$.
\end{abstract}

DOI: 10.1103/PhysRevE.64.041304

\section{INTRODUCTION}

Gas-fluidized beds are widely used in industry, and their properties have been the subject of research for some decades. An important issue is to understand the settling rate of the particles when the fluidizing gas is suddenly stopped. The problem is analogous to that of the sedimentation of suspensions of solid particles in a liquid, which, in spite of having a long and well-deserved history for its ubiquitous applications [1], is not yet well understood. For a multiparticle system at low solid volume fractions $(\phi)$, long ranged hydrodynamic interactions hinder sedimentation [2]. At higher values of $\phi$ the presence of a fluid backflow arising from the sedimenting particles, interparticle collisions, and crowding become increasingly important [3]. The relationship between the settling velocity in sedimentation $v_{s}$ and the solid volume fraction $\phi$ for a homogeneous gas-fluidized powder of noncohesive spheres was well described by the phenomenological Richardson-Zaki law [4]

$$
\frac{v_{s}}{v_{p 0}}=(1-\phi)^{n} .
$$

Here $v_{p 0}$ is the Stokes settling velocity of a single particle, $v_{p 0}=(2 / 9)\left(\rho_{p}-\rho_{f}\right) a^{2} g / \mu$ in the laminar regime, where $\rho_{p}$ is the particle density, $\rho_{f}$ is the gas density, $g$ is the gravity field, $a$ is the particle radius, $\mu$ is the viscosity of the gas, and $n$ is a parameter of order 5 in the small Reynolds number limit. Buscall et al. [5] reported on measurements of the mean fall velocity of sedimenting polystyrene spheres in water in a small Reynolds number range over the whole range of values of $\phi$ which fitted to the Richardson-Zaki law for $n=5.5$. Theoretical considerations by Batchelor [6] led to $v_{s} / v_{p 0} \simeq 1-5.6 \phi[7]$ for very diluted suspensions of nonBrownian monodisperse hard spheres in the limit of small Reynolds numbers. Thus the sedimentation velocity predicted by Eq. (1) conformed with the dilute limit of Batchelor for $n=5$.6. The simplicity of the Richardson-Zaki equation, although satisfying from a practical point of view,
PACS number(s): 47.70. $-\mathrm{n}$, 47.55.Kf, 47.55.Mh

lacked any theoretical justification at high values of $\phi$. Mills and Snabre [8] theoretically derived the settling velocity for a system of concentrated noncohesive spheres from the energy balance during the sedimentation process. They used mean field and scaling arguments to arrive at the expression

$$
\frac{v_{s}}{v_{p 0}}=\frac{1-\phi}{1+k \phi /(1-\phi)^{3}},
$$

and reported on excellent agreement with experimental data by choosing the parameter $k=4.6$ to match Eq. (2) in the diluted limit $\left[v_{s} / v_{p 0}=1-(k+1) \phi\right]$ to Batchelor's result. Equation (2) yields, in the relevant range $\phi<\sim 0.6$, results almost identical to those of the empirical Richardson-Zaki equation.

When the particle size is smaller than some critical value, the gravity is dominated by interparticle forces. This makes an understanding of the settling process more complicated [22]. In situations dealing with the flow of the bulk powder, it is generally observed that the tendency of particles to aggregate leads to a substantial departure from the behavior of free-flowing particulate systems [9-11]. From numerical simulations and experiments Nase et al. [11] found that the static angle of repose of a granular heap did not depend on the particle size if the particle weight $W$ exceeded the interparticle adhesion force $F_{t}$, but increased markedly as $F_{t} / W \equiv \mathrm{Bo}_{g}$ was increased above 1 . As with the static heap angle, a sharp decrease was observed to occur in the discharge rate through a hopper when $\mathrm{Bo}_{g}$ was increased above 1. In that work cohesion arose from capillary forces, and the nondimensonal number $\mathrm{Bo}_{g}$ was reminiscent of the Bond number in fluid mechanics. For this reason it was referred to as the granular Bond number [11]. In the case of dry fine particles (particle size $<\sim 30 \mu \mathrm{m}$ ) the van der Waals attractive force is dominant and may exceed the particle weight $[14,15]$ by several orders of magnitude. Numerical simulations and experiments [12] revealed the important role of the ratio of this force to particle weight (for which, hereafter, we 
keep the denomination of the granular Bond number) on the packing of fine particles. The solid volume fraction of the packing was shown to decrease as $\mathrm{Bo}_{g}$ was increased above 1 . However, the decrease of $\phi$ became significant only when $\mathrm{Bo}_{g} \geqslant 10$. For $\mathrm{Bo}_{g} \leqslant 10$, the value $\phi$ closely approached 0.64 , which is the limit of random close packing of noncohesive spheres [16].

The agglomeration of fine particles in fluidized beds due to attractive van der Waals force is a well known phenomenon [17-19], but the effect of aggregation on sedimentation has scarcely been addressed in the literature. Lettieri et al. [13] reported on deviations from the Richardson-Zaki law in the settling of fcc catalysts powders (average particle size $50 \mu \mathrm{m})$ when contact forces between particles were increased by the presence of potassium acetate. Allain et al. $[20,21]$ showed that the settling of a fractured gelled suspension of calcium carbonate particles (particle diameter $0.07 \mu \mathrm{m})$ is well described by a modified Richardson Zaki correlation law $v_{s}=0.33\left(1-\phi / \phi^{*}\right)^{5.1 \pm 0.4}$, where $\phi^{*}$ is the particle volume fraction inside the sediment. Elsewhere we proposed [22] a similar equation to account for the effect of aggregation in gas-fluidized beds. Assuming that the aggregates behave like hard spheres with a hydrodynamic radius equal to their radius of gyration $R$, we arrived at

$$
\frac{v_{s}}{v_{p 0}}=\frac{N}{\kappa}\left(1-\phi \frac{\kappa^{3}}{N}\right)^{n}
$$

where $N$ is the number of aggregated particles and $\kappa \equiv R / a$.

Equation (3) is valid as long as aggregates can be considered as individuals. We shared elsewhere [23] that a stable gas-fluidized bed presents two distinct regimes of dynamical behavior. For solid volume fractions above a certain critical value $\phi_{c}$ the particles in the fluidized bed remain static, sustained in part by enduring contacts. The bed then behaves as a weak solid [24]. For example, when it is tilted the free surface remains stable because of the existence of a certain mechanical strength. In the second regime, for $\phi<\phi_{c}$, permanent contacts no longer exist, the mechanical strength of the bed vanishes, and stresses are carried by interstitial gas and aggregate collisions due to fluctuations in their motion. The powder then takes on many of the properties of a fluid, its upper surface remaining horizontal when the container is tilted. The boundary between both regimes can be found by measuring the tensile strength of the fluidized powder, that becomes zero when the bed enters the fluidlike regime [23].

We will report measurements of the settling velocity on a set of cohesive powders differing in particle surface structure and particle size. The aggregation of these cohesive particles is investigated through the coupling between aggregation and sedimentation. Fitting the experimental results to Eq. (3), we will obtain the number of particles per aggregate as well as the aggregate size as a function of the granular Bond number. We will show that at a critical value of the solid volume fraction the settling rate slows down because the bed enters a solidlike regime where enduring interparticle contacts hinder sedimentation. Thus our experiments will also serve to determine the transition from fluidlike to solidlike behavior.

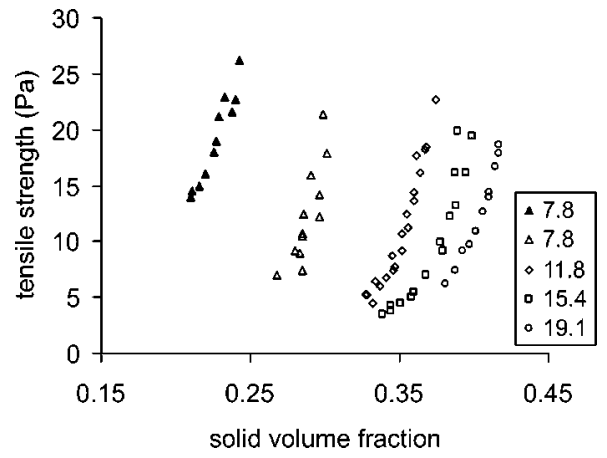

FIG. 1. Tensile strength measured by a powder bed technique [25] for a set of toners used as a function of the solid volume fraction (particle size is indicated in $\mu \mathrm{m}$ ). Open symbols correspond to $32 \%$ SAC and solid symbols to $8 \%$ SAC. The estimated vertical error bar is $2 \mathrm{~Pa}$.

\section{MATERIALS}

The powders used in this study were made from the random copolymer styrene $n$-butylmethacrylate (particle density $1135 \mathrm{~kg} / \mathrm{m}^{3}$ ). The particles were produced by an attrition process followed by size classification using a cyclone separator apparatus. By this technique four sets of powders were produced whose particles are of volume average diameters $19.1,15.4,11.8$, and $7.8 \mu \mathrm{m}$. Particles are irregularly shaped, since they are formed by a grinding process and are rather monodisperse (the typical standard deviation from the average is $1 \mu \mathrm{m}$ ). These powders were blended with a surface additive such as Aerosil ${ }^{R}$ (nanoparticles of fumed silica), which is well known for its ability to control interparticle cohesive forces [14]. The present paper includes powders with additive concentrations of $8 \%$ and $32 \%$ surface area coverage (SAC), making a matrix of eight powders covering a range of four particle sizes and two levels of cohesivity. Measurements on the tensile strength of these powders are reported elsewhere $[25,26]$. In Fig. 1 we present experimental results of the tensile strength versus the solid volume fraction for a set of powders used in the present study. A marked trend of the packing fraction with particle size is found. Powders that flow well (higher particle size) pack in closer structures, while as the particle size decreases the powder flowability decreases and particles pack in very open structures. The effect of the fumed silica on the packing is also clearly seen in Fig. 1. Increasing SAC causes a better flow of the powder, and results in a closer packing. The effect of the additive is more pronounced for finer powders [26], since in this case the bare particles are very cohesive, i.e., the interparticle van der Waals force is much larger than the weight of the particles. From the measured macroscopic stresses we have estimated $[14,26]$ the interparticle contact forces under the assumption of a uniform distribution of stresses inside the material. Results of the estimated adhesion force per contact $F_{t}$, as a function of the estimated load force per contact $F_{c}$ in the range of low load forces, are drawn in Fig. 2. In the range of low stresses, $F_{t}$ does not depend on particle size, implying that only stresses in the contact region are important for the contact forces. The addition of fumed silica nanoparticles results in an increase of 


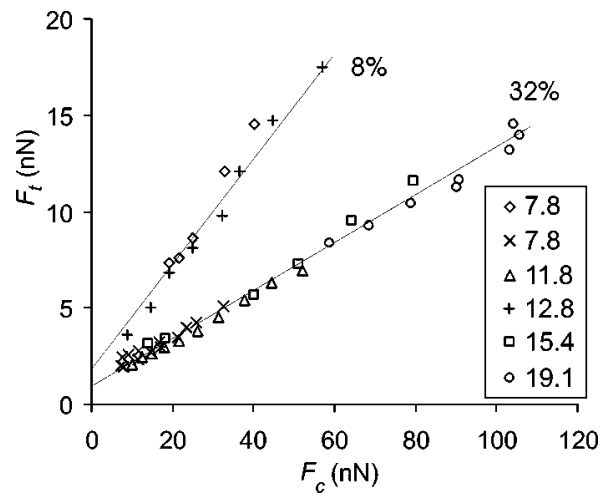

FIG. 2. Estimated adhesion force per contact $F_{t}$ as a function of the estimated load for per contact $F_{c}$ from measured bulk stresses $[14,26]$ (particle size is indicated in $\mu \mathrm{m}$ and the surface silica coverage is indicated for each group of data). The estimated error bar is $1 \mathrm{nN}$.

the contact hardness, because the particles are made of a harder material than polymer. The increase in hardness cause a significant reduction of the slope $F_{t}-F_{c}$ (see Fig. 2), as predicted by theory [26].

The additive could also play a role in the fluidized regime, where the load force on the contacts due to gravity is removed by the gas flow. The reason for this is the reduction of the size of the contacts by the increase of surface rugosity. However, as can be observed from Fig. 2, the reduction in the adhesion force extrapolated to $F_{c}=0$, due to the increase of surface silica coverage, is quite small (the measured reduction is of the order of the error bar of the measurements). From this result it can be anticipated that the initial settling velocity of the fluidized bed will not be very sensitive to the surface properties of our dry fine powders. This will be confirmed by our experimental work.

For comparison, spherical soda lime glass beads (particle size $\sim 40 \pm 10 \mu \mathrm{m}$ and density $2500 \mathrm{~kg} / \mathrm{m}^{3}$ ) were also tested. In spite of their large density these particles could be fluidized. Larger glass beads, although available, did not fluidize homogeneously [27], and their settling velocity could not be measured.

\section{EXPERIMENTAL SETUP}

The experimental setup is shown in Fig. 3. The powder sample is held in a $4.45-\mathrm{cm}$ diameter, $17-\mathrm{cm}$ height cylinder made of polycarbonate, the base of which is a sintered metal filter of $5-\mu \mathrm{m}$ pore size, and of $1.5-\mathrm{mm}$ width. A dry nitrogen tank, furnished with a mass flow controller, supplies an adjustable gas flow. By using dry nitrogen, the effects of moisture on the interparticle adhesion are minimized. All the measurements start by driving the powder into the freely bubbling phase. Once the powder is in a bubbling phase the fluidizing gas is set down to a given value below the minimum bubbling point, and the powder settles to a reproducible and stable fluidized state. The solid volume fraction of the system is conveniently varied by controlling the gas flow rate. The bed height is read from an ultrasonic sensor, and from these data the solid volume fraction is calculated. Once

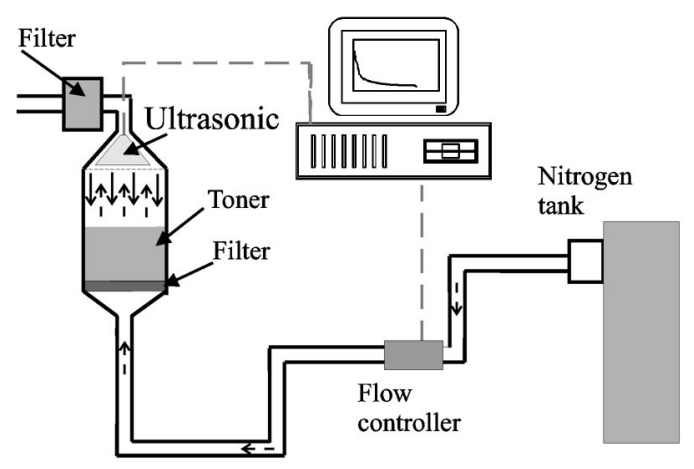

FIG. 3. Experimental setup. Immediately after the gas flow is turned off the bed height is continuously acquired by the ultrasonic sensor placed at the top of the bed.

the powder reaches a steady state for a given fluidization velocity, the gas flow is abruptly reduced to zero and the powder settles down. During the sedimentation process the bed height is continuously acquired by the ultrasonic sensor. Figure 4 displays a typical sedimentation curve. The head of the bed falls initially at a constant velocity, as long as aggregates settle individually. However, when the bed reaches a critical height (indicated by the arrow in the plot), the settling process is hindered by stresses carried by enduring interparticle contacts. At this point the bed enters the solidlike regime. The initial settling velocity of the head of the bed $v_{s}$ is taken as the initial slope of the sedimentation curve. As we showed elsewhere [22] we find that $v_{s}$ is approximately equal to the gas velocity $v_{g}$. This should be expected, since the particles in the fluidization phase are moving down from the gas frame at a velocity equal to the superficial gas velocity. Hence when the gas supply is suddenly stopped, the initial settling velocity of the particles must coincide with the gas velocity.

\section{EXPERIMENTAL RESULTS}

\section{A. Effect of surface additives}

In Fig. 5 results of the initial settling velocity are plotted against the initial solid volume fraction of the fluidized pow-

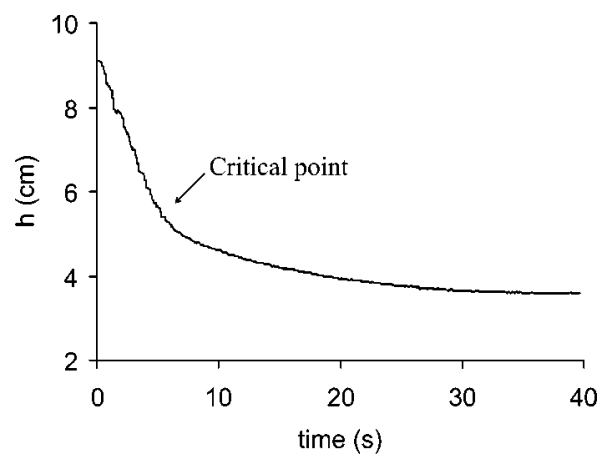

FIG. 4. Powder bed height $(7.8-\mu \mathrm{m}$ particle size powder, $32 \%$ SAC) as a function of time after the gas supply is suddenly stopped for an initial superficial gas velocity of $0.56 \mathrm{~cm} / \mathrm{s}$. The height is measured by means of an ultrasonic device, having a typical indeterminacy of $0.05 \mathrm{~cm}$. 


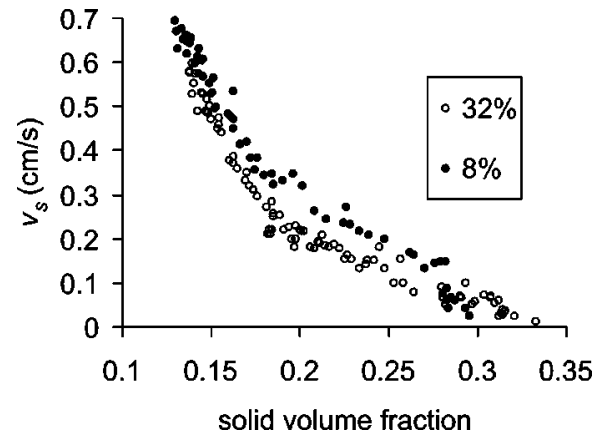

FIG. 5. Initial settling velocity of the head of the powder bed as a function of the solid volume fraction of the powder before the gas supply was stopped for powders of the same particle size $(11.8 \mu \mathrm{m})$ but different surface additive coverage. The estimated error bar is of the order of symbol size.

der. Results for powders with the same particle size (11.8 $\mu \mathrm{m})$ but different values of surface additive coverage are compared. It is observed that the settling velocity increases slightly when the SAC value is reduced from $32 \%$ to $8 \%$. The same is obtained for other powders of different particle size when the SAC value is reduced. This result is consistent with the formation of aggregates due to interparticle attractive forces. As the additive surface coverage is decreased, the interparticle adhesion force increases and particles tend to be more aggregated. Larger aggregates would give larger values of the settling velocity, as we obtain experimentally. However, the increase of interparticle adhesion in the absence of an external load (fluidized state) is very small (see Fig. 2). Accordingly, the effect on the increase of the settling velocity is not pronounced.

\section{B. Effect of particle size}

In Fig. 6 the initial settling velocities are represented against the solid volume fraction of the fluidized powder for the set of powders with $32 \%$ SAC and different particle size. We observe that for a given value of $\phi$ the initial settling velocity increases markedly as the particle size is increased. The predicted curves from Eq. (1) for the settling of indi-

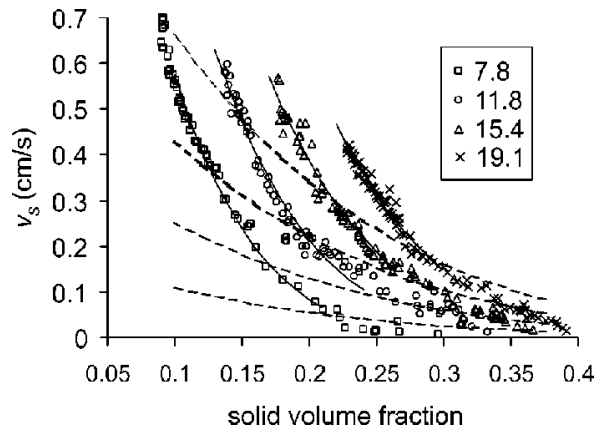

FIG. 6. Initial settling velocity of the head of the powder bed as a function of the solid volume fraction of the powder before the gas supply was stopped for the set of powders used (particle size is indicated in $\mu \mathrm{m}$ ). Predicted curves from Eq. (1) (- - -) and best fit curves from Eq. (3) (-) in the fluidlike regime are also shown. The estimated error bar is of the order of symbol size.
TABLE I. Critical solid volume fraction predicted from the settling experiments for the transition from the fluidlike to solidlike fluidization regime as a function of the particle diameter $(2 a)$ for $32 \%$ SAC.

\begin{tabular}{cc}
\hline \hline $2 a(\mu \mathrm{m})$ & $\phi_{c}$ \\
\hline 7.8 & 0.22 \\
11.8 & 0.26 \\
15.4 & 0.28 \\
19.1 & 0.29 \\
\hline
\end{tabular}

vidual particles are also plotted in Fig. 6. Equation (1) underestimates experimental results for $\phi<\phi_{c}$, and overestimates them for $\phi>\phi_{c}$. Table I shows the values of $\phi_{c}$ as a function of particle size. In a single settling experiment we observe that the sedimentation velocity decreases at $\phi$ $=\phi_{c}$. Thus at $\phi=\phi_{c}$ the transition form fluidlike to solidlike behavior is expected. For $\phi>\phi_{c}$ the bed would have a mechanical strength caused by permanent particle contacts that hinder the sedimentation process. Conversely, for $\phi$ $<\phi_{c}$, particles aggregate and display a collisional dynamics. The effect of aggregation in this regime is an increase of the settling velocity over the predicted value by Eq. (1), where particles are considered free floating individuals. Although the measurements of the tensile strength $\sigma_{t}$ near the solidfluid transition are quite difficult, the extrapolation of our experimental results (Fig. 1) to $\sigma_{t}=0$ gives a similar trend of the critical solid volume fraction $\phi_{c}$ with particle size (see Table I). Elsewhere we reported [22] results on the settling velocity obtained for the commercial toner Canon CLC700 (particle size $8.53 \pm 2.32 \mu \mathrm{m}$ ). According to that work the value of $\phi_{c}$ for the powder would be $\phi_{c} \simeq 0.28$, which matches the value obtained from independent measurements on the diffusion coefficient, tensile strength, and maximum stable angle of inclination [23].

The best fit curves from Eq. (3), where the aggregation of particles is taken into account, to the experimental results in the fluidlike regime are also plotted in Fig. 6. Table II shows the best fitting parameters to the experimental data. As should be expected, the number of aggregated particles $N$ and the ratio of the aggregate radius to particle radius $\kappa$ $=R / a$ increase as the particle size is decreased. Interestingly, $R$ shows a weak dependence on the particle size, being around $40 \mu \mathrm{m}$ for all powders investigated. The scaling of $\kappa$ with the number of aggregated particles can be used to determine the fractal dimension $D$ of the aggregates,

TABLE II. Average number of aggregated particles $N$, ratio of aggregate radius $R$ to particle radius $a$ and fractal dimension $D$ $=\ln N / \ln (R / a)$ for particle aggregates (32\% SAC).

\begin{tabular}{cccc}
\hline \hline $2 a(\mu \mathrm{m})$ & $N$ & $R / a$ & $D$ \\
\hline 7.8 & 63 & 5.218 & 2.523 \\
11.8 & 23.7 & 3.549 & 2.512 \\
15.4 & 12.4 & 2.724 & 2.499 \\
19.1 & 9.6 & 2.448 & 2.508 \\
\hline \hline
\end{tabular}


$D=\ln N / \ln \kappa$. We obtain $D \simeq 2.5$ independently of the particle size (see Table II). This is approximately the value of $D$ given by the diffusion-limited aggregation model introduced by Witten and Sander [28] in three-dimensional systems, where self-similar fractal ramified patterns grow via irreversible sticking of particles in a random motion. Indeed, fine particles display a diffusive dynamics in the fluidized bed [23], and stick to aggregates due to the domination of interparticle adhesion force over particle weight. Thus aggregates should appear as branched structures whose growth is controlled by a diffusive process.

\section{Visualization of aggregates}

To further investigate the aggregation properties of fine particles, we have visualized the structure of the fluidized bed. We made use of the great tendency of powder particles to adhere to a paper surface. A paper card was carefully lowered edgewise into the fluidized bed, so as to cleave it perpendicular to the gas flow path. Then, on carefully and slowly withdrawing the card, it was found to carry a replica of the fluidized powder. Although this technique introduces an unavoidable perturbation, we expect it to be small due to the low values of the extracting velocity and solid volume fraction. Furthermore, it has been proven elsewhere [10,22] to be very effective in yielding a good replica of the powder. Figures 7(a) and 7(b) show sample pictures from the optical microscope. As anticipated, we observe that particles are aggregated, forming branched structures. Furthermore, in the case of the larger particles, we can distinguish that aggregates are formed by a number of the order of ten particles, which agrees with our estimation from the settling experiment (see Table II). It is also apparent from Fig. 7(b) that when the particle size is reduced the number of aggregated particles increases.

\section{DISCUSSION}

Cohesion between particles may arise from a variety of sources. Dispersion or van der Walls forces, electrostatic forces, and capillary forces are regarded as the main forces in particle adhesion. We have minimized capillary and electrostatic forces, working with uncharged particles in an ambient of dry nitrogen gas. Consequently, van der Waals forces are dominant between our dry fine particles. These forces have their origin in the interaction of molecules through fluctuating dipole-dipole fields. Summing up all the interactions between two spherical and rigid particles in contact with diameters $d_{1}$ and $d_{2}$ leads to an attractive force [29]

$$
F_{v d W}=\frac{A}{12 z_{0}^{2}}\left(\frac{d_{1} d_{2}}{d_{1}+d_{2}}\right),
$$

where $A$ is the Hamaker constant and $z_{0}$ is the distance of closest approach between two molecules which is about $4 \AA$ [30]. At very small loads, the deformation of the contact is negligible, and Eq. (4) can be taken approximately as the interparticle adhesion force. Most powder particles have a rather rough surface with many asperities [31] of typical
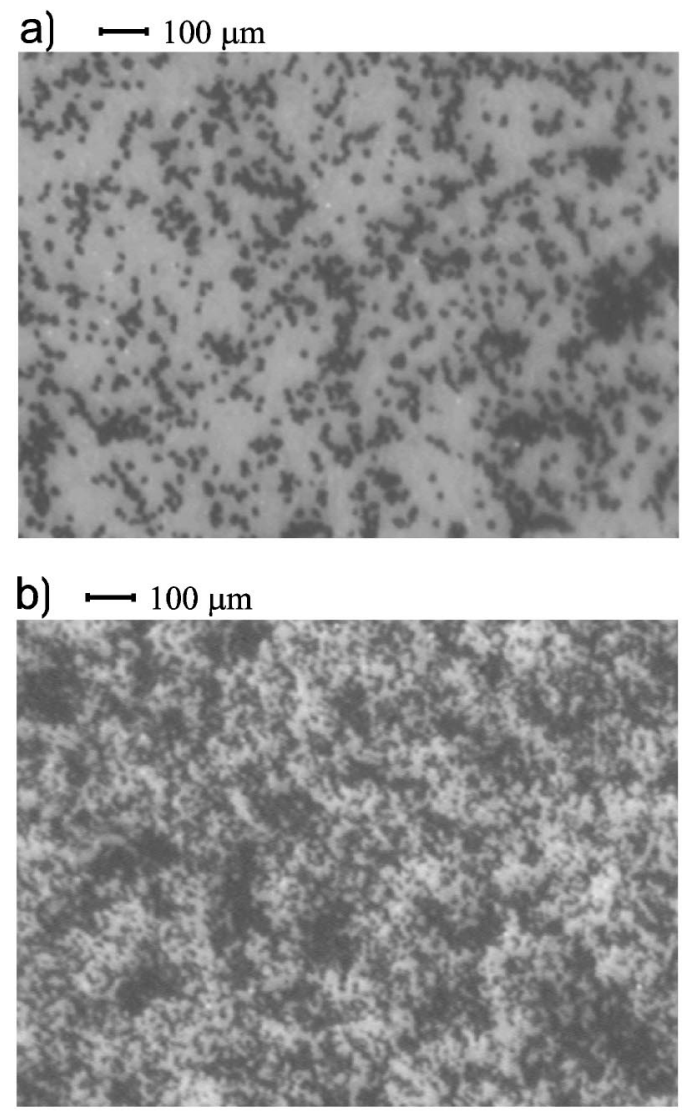

FIG. 7. (a) Picture taken from the optical microscope of particle aggregates of a gas-fluidized bed of particles of size $19.1 \mu \mathrm{m}, 32 \%$ SAC. (b) Same as (a), but for particles of size $7.8 \mu \mathrm{m}$.

sizes often not larger than $0.2 \mu \mathrm{m}$ [32]. Thus the effective contact area is given by the asperities at contact, and the interparticle attractive force can be approximately obtained [33] inserting the typical diameter of the asperities $d_{a}$ in Eq. (4). One of the reasons for which silica additive is used in xerographic toners is to increase surface rugosity, thus decreasing the typical size of the asperities at contact. From typical scanning electron microscopy micrographs of xerographic toner particles, it is observed that the additive particles are distributed in agglomerates with estimated diameters of $40 \pm 5 \mathrm{~nm}$ [34]. If the surface coverage of additives is $32 \%$, we would expect that a large part of contacts would be silica to silica. Thus we use the average diameter of silica aggregates as the diameter of the surface asperities, and $A$ $\approx 1.5 \times 10^{-19} \mathrm{~J}$ for silica [35] is inserted into Eq. (4). On the other hand, for only a $8 \%$ of surface coverage of silica the contacts are most likely to occur between the polymer surfaces of the particles. In such a case $A \approx 0.65 \times 10^{-19} \mathrm{~J}[35]$, and we will take $d_{a} \simeq 0.2 \mu \mathrm{m}$. According to these numbers, Eq. (4) yields $F_{v d W} \simeq 1.6 \mathrm{nN}$ for $32 \% \mathrm{SAC}$, and $F_{v d W}$ $\simeq 3.4 \mathrm{nN}$ for $8 \%$ SAC. Note that these results agree, within experimental scatter, with extrapolation to zero load of the estimated interparticle adhesion force from the measured bulk stresses (see Fig. 2). Thus the change in the interparticle adhesion force at zero load (fluidized state), when the surface additive coverage is reduced, is quite small. As long as the 


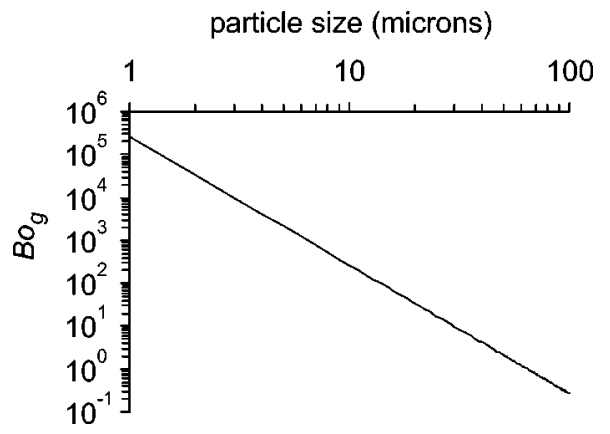

FIG. 8. Theoretical granular Bond number calculated as the ratio of interparticle attractive van der Waals force [Eq. (4) with $A$ $=1.5 \times 10^{-19} \mathrm{~J}, d_{1}=d_{2}=0.05 \mu \mathrm{m}$, and $\left.z_{0}=4 \AA\right]$ to particle weight (assuming perfect spherical particles), as a function of particle diameter.

particle size is held constant, the decrease in the $\mathrm{Bo}_{g}$ number is also small. Thus the average number of aggregated particles is expected to increase only slightly. This would explain why the effect of reducing the SAC value on the initial settling velocity of the powder is not marked (Fig. 5).

Indeed the main effect on the settling velocity is caused by a change in particle size (Fig. 6). In Fig. 8, the calculated $\mathrm{Bo}_{g}$, assuming contact between silica aggregates, is shown as a function of particle diameter. As can be seen, $\mathrm{Bo}_{g}$ is very sensitive to the particle size, increasing in more than two orders of magnitude when particle size is decreased from $19.1 \mu \mathrm{m}\left(\mathrm{Bo}_{g}=39\right)$ to $7.8 \mu \mathrm{m}\left(\mathrm{Bo}_{g}=5700\right)$. Thus the average number of aggregated particles should increase markedly as the particle size is reduced. In Fig. 9 we represent the fitting parameters $N$ and $\kappa=R / a$ as a function of the granular Bond number. Results for $N$ and $\kappa$ for commercial toner Canon CLC700, reported elsewhere [22], are also plotted in Fig. 9. We see from this figure that $N$ and $\kappa$ increase following a potential law as the granular Bond number increases. Extrapolating these results we would predict that $N \simeq 1$ and $R / a \simeq 1$ for $\mathrm{Bo}_{g} \simeq 1$.

To make clear the fundamental role of particle size in the aggregation of gas-fluidized particles we have carried out the

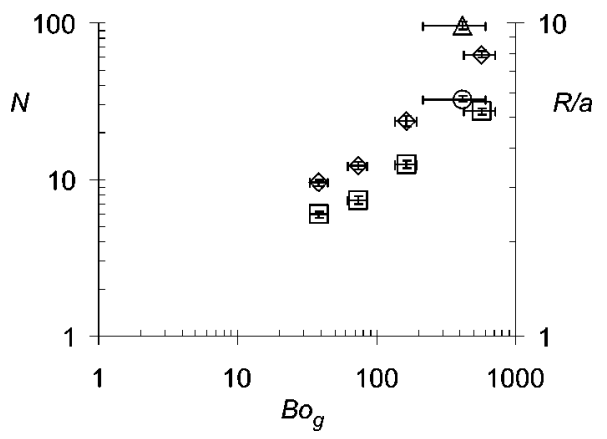

FIG. 9. Number of aggregated particles $(N: \diamond)$ and ratio of aggregate size to particle size $(R / a: \square)$ calculated from the fit of Eq. (3) to experimental data (Fig. 6) as a function of the granular Bond number of toner particles. Results from experiments on toner Canon CLC700 $(N: \triangle$ and $R / a: \bigcirc)$ reported elsewhere [22] are also shown. settling experiment with dry glass beads (particle size $\sim 40$ $\pm 10 \mu \mathrm{m}$ and density $2500 \mathrm{~kg} / \mathrm{m}^{3}$ ). Taking $A \simeq 10^{-19} \mathrm{~J}$ and $d_{a} \simeq 0.2 \mu \mathrm{m}$, the granular Bond number is $\sim 6$, and therefore we should expect almost no aggregation. Accordingly their settling velocity should be well described by the Richardson-Zaki law for individual particles. The measured solid volume fraction of the settled beads is $\phi \simeq 0.51$. The bed was fluidized and expanded up to $\phi \simeq 0.44 \pm 0.01$. Then the gas flow was suddenly stopped. The average value of the ratio of the measured sedimentation velocity to the settling velocity of an individual particle was $v_{s} / v_{p 0} \simeq 0.037$ \pm 0.005 . On the other hand, the predicted value by the Richardson-Zaki law for noncohesive spherical particles [Eq. (1)] is $v_{s} / v_{p 0} \simeq(1-0.44)^{5.6} \simeq 0.039$, which is in agreement with the experimental result.

In sum these results suggest a change of behavior, from free-flowing to aggregated particulate systems, for $\mathrm{Bo}_{g}>$ $\sim 1$, much in the same way as already found when cohesion was originated from capillary forces [11]. Our results are also in agreement with computer simulation results that predict a decrease in the packing fraction for $\mathrm{Bo}_{g}>\sim 1$ [12].

\section{CONCLUSION}

The strong interparticle van der Waals force existing between fine particles induce aggregation, and plays a fundamental role in the behavior of gas-fluidized beds of fine powders. This force depends on the surface structure of the particles, i.e., surface density and size distribution of asperities. We have used a set of fine powders differing in particle size and surface additive coverage. Settling experiments have served to identify the transition from a fluidlike to a solidlike fluidized regime at a given value of the solid volume fraction. This transition has been confirmed by measurements of the mechanical strength and diffusivity of the bed in the fluidized state. Our experimental results on the collapse of gas-fluidized beds in the fluid-like regime can be explained by a modified Richardson-Zaki law that takes into account the agglomeration of fine particles. In this way we have determined the average number of particles per aggregate $N$ and the ratio of the aggregate radius to particle radius $\kappa$, which result to be directly linked to the granular Bondnumber (the ratio of the interparticle adhesive force to the particle weight). The trend followed by the fitting parameters $N$ and $R / a$ has been confirmed by direct visualization of the aggregates and suggests that particles aggregate when $\mathrm{Bo}_{g}$ $>\sim 1$. Aggregates are found to have a fractal structure that can be approximately described by the DLA model.

\section{ACKNOWLEDGMENTS}

This research was supported by the Xerox Foundation, Spanish Government Agency Ministerio de Ciencia y Tecnologia (DGES) under Contract No. BMF2000-1056, and NATO Grant LINKAGE PST.CLG.976575. 
[1] W. Russel, D. Saville, and W. Schowalter, Colloidal Dispersions (Cambridge University Press, Cambridge, 1995).

[2] A. J. C. Ladd, Phys. Fluids 9, 491 (1997).

[3] P. N. Segre, F. Liu, P. Umbanhowar, and D. A. Weitz, Nature (London) 409, 594 (2001); P. N. Segre, E. Herbolzheimer, and P. M. Chaikin, Phys. Rev. Lett. 79, 2574 (1997).

[4] J. F. Richardson and W. N. Zaki, Trans. Inst. Chem. Eng. 32, 35 (1954).

[5] R. Buscall, J. W. Godwin, R. H. Otewill, and T. F. Tadros, J. Colloid Interface Sci. 85, 78 (1982).

[6] G. K. Batchelor, J. Fluid Mech. 119, 379 (1982).

[7] G. K. Batchelor and C.-S. Wen, J. Fluid Mech. 124, 495 (1982).

[8] P. Mills and P. Snabre, Europhys. Lett. 25, 651 (1994).

[9] A. Castellanos, J. M. Valverde, A. T. Perez, A. Ramos, and P. K. Watson, Phys. Rev. Lett. 82, 1156 (1999).

[10] J. M. Valverde, A. Castellanos, A. Ramos, and P. K. Watson, Phys. Rev. E 62, 6851 (2000).

[11] S. T. Nase, W. L. Vargas, A. A. Abatan, and J. J. McCarthy, Powder Technol. 116, 214 (2001).

[12] R. Y. Yang, R. P. Zou, and A. B. Yu, Phys. Rev. E 62, 3900 (2000).

[13] P. Lettieri, J. G. Yates, and D. Newton, Powder Technol. 110, 117 (2000).

[14] J. M. Valverde, A. Ramos, A. Castellanos, and P. K. Watson, Powder Technol. 97, 237 (1998).

[15] J. P. K. Seville, C. D. Willett, and P. C. Knight, Powder Technol. 113, 261 (2000).

[16] J. Berryman, Phys. Rev. A 27, 1053 (1983).

[17] N. Hawley, J. Geophys. Res. 87, 9489 (1982).

[18] A. F. Fortes, P. Caldas, and J. V. Gallo, Powder Technol. 98, 201 (1998).
[19] T. Zhou and L. Hongzhong, Powder Technol. 101, 57 (1999).

[20] C. Allain, M. Cloitre, and M. Wafra, Phys. Rev. Lett. 74, 1478 (1995).

[21] C. Allain, M. Cloitre, and M. Wafra, in Physical Chemistry of Colloids and Interfaces in Oil Production, edited by H. Toulhoat and J. Lecourtier (Technip, Paris, 1992), p. 259.

[22] J. M. Valverde, M. A. S. Quintanilla, A. Castellanos, and P. Mills, Europhys. Lett. 54, 329 (2001).

[23] J. M. Valverde, A. Castellanos, and M. A. S. Quintanilla, Phys. Rev. Lett. 86, 3020 (2001).

[24] N. Menon and D. J. Durian, Phys. Rev. Lett. 79, 3407 (1997).

[25] J. M. Valverde, A. Castellanos, A. Ramos, A. T. Perez, M. A. Morgan, and P. K. Watson, Rev. Sci. Instrum. 71, 2791 (2000).

[26] P. K. Watson, J. M. Valverde, and A. Castellanos, Powder Technol. 115, 44 (2001); J. M. Valverde, A. Castellanos, and P. K. Watson, Powder Technol. 118, 240 (2001).

[27] K. Rietema, The Dynamics of Fine Powders (Elsevier, London 1991).

[28] T. A. Witten and L. M. Sander, Phys. Rev. Lett. 47, 1400 (1981).

[29] H. C. Hamaker, Physica (Amsterdam) 4, 1058 (1937).

[30] H. Krupp, Adv. Colloid Interface Sci. 1, 111 (1967).

[31] T. G. Mason, A. J. Levine, D. Ertas, and T. C. Halsey, Phys. Rev. E 60, 5944 (2000).

[32] L. Massimilla and G. Donsi, Powder Technol. 15, 253 (1976).

[33] A. J. Forsyth and M. J. Rhodes, J. Colloid Interface Sci. 223, 133 (2000).

[34] M. L. Ott and H. A. Mizes, Colloids Surf., A 87, 245 (1994).

[35] S. Ross and I. D. Morrison, Colloidal Systems and Interfaces (Wiley-Interscience, New York, 1988). 